\title{
Braucht es spezielle Weiterbildner in der Chirurgie?
}

\author{
Urs Neffa, Brigitte Muff ${ }^{b}$ \\ a Verantwortlicher Leitender \\ Arzt für Weiterbildung \\ b Chefärztin Chirurgie und \\ Vorstandsmitglied Schweize- \\ risches Institut für ärztliche \\ Fort- und Weiterbildung \\ SIWF
}

Korrespondenz: PD Dr. med. Urs Neff

Tannerberg 11 CH-8200 Schaffhausen Tel. 0526257000

urs.neff[at]gmx.ch

PD Dr. med. Brigitte Muff Spital Bülach CH-8180 Bülach Tel. 0448632362

\author{
Eine qualifizierte Weiterbildung ist nicht nur \\ die beste Nachwuchswerbung, \\ sondern folgt dem genuinen Auftrag, \\ die Patienten qualifiziert zu behandeln. \\ (Carsten J. Krones. \\ Der Chirurg. BDC. 3.2009:117)
}

Weiterbildung (WB) in der Medizin, speziell Chirurgie, liegt zur Zeit aus zwei Gründen im Brennpunkt des Interesses: einerseits wegen der Unsicherheit der Finanzierung mit der Einführung von Fallpauschalen im Jahre 2012, andererseits wegen des Mangels an strukturierter WB und des fehlenden Nachwuchses [1].

Der zunehmende Druck auf eine effiziente WB in der Chirurgie hat verschiedene Ursachen:

- Der ökonomische Druck auf die Spitäler und deren Kader, wirtschaftlicher zu denken, wird immer stärker. Die Entwicklung im Gesundheitswesen wird zunehmend von wirtschaftlichen Interessen [2, 3], den Marktkräften und ökonomischen Zwängen beeinflusst. Ökonomisch bedingte Restriktionen führen dazu, die Prioritäten eindeutig zugunsten der Dienstleistung und auf Kosten der Weiterbildung zu verlagern.

- Erhöhte Forderungen an die Weiterbildung durch die Standesorganisation SIWF und das Bundesamt für Gesundheit (BAG). Entsprechend dem heutigen Zeitgeist hat die Ärztekammer der FMH im Dezember 2008 acht zentrale Zielsetzungen für die Legislaturperiode 2008 bis 2012 gefasst. Darunter fällt auch die Sicherung der ärztlichen Berufsbildung und Förderung des beruflichen Nachwuchses. Das BAG seinerseits fordert die Fachgesellschaften auf, ihre WB anhand des Standards der «World Federation of Medical Education» kritisch zu beleuchten. Dazu gehören dezentrale praktische WB an und mit dem Patienten, Stärkung der Lehrprozesse, Steigerung der Effizienz in der ärztlichen WB durch strukturierte, in eigens der WB vorbehaltenen Zeiten [4].

- Das Berufsbild und gleichzeitig die Attraktivität der Medizin insbesondere der Chirurgie hat sich für Studienabgänger wesentlich gewandelt. Nach H. Bauer [1] werden dafür mehrere Gründe verantwortlich gemacht, so etwa hohe Belastung im Vergleich zu anderen Fächern, ständig steigende Arbeitsverdichtung, ökonomischer Druck mit kürzerer Patientenverweildauer, zermürbende

\section{A-t-on besoin de formateurs particuliers en chirurgie?}

En 2008, un poste de formateur responsable de la formation postgraduée a été créé au sein du service de chirurgie de l'hôpital de Bülach dans le cadre d'un projet pilote. Bénéficiant désormais d'un accompagnement spécialisé, les nouveaux médecins-assistants peuvent aborder plus sereinement leur entrée dans le monde du travail. Cet accompagnement permet également de répondre à des questions d'ordre technique mais aussi éthique et scientifique qui se posent fréquemment en début de formation postgraduée. De notre point de vue, le bilan de ce projet pilote s'avère positif et ce poste peut être considéré comme le pendant de la formation pratique complémentaire qui existe déjà dans le domaine des soins. Le titulaire du poste doit avant tout être au bénéfice d'une riche expérience mais n'a pas besoin d'avoir suivi de formation particulière ou de disposer d'un Master of medical education (MME) par exemple.

Bürokratie mit grösser werdender Flut von Anfragen, Mangel an Förderung und Mentoring, wenig familienfreundliche Strukturen, sinkendes Sozialprestige und unsichere Zukunftsperspektiven. In Deutschland wollen nur noch 6\% der jungen Ärztinnen und Ärzte den Weg in die Chirurgie wählen.

\section{Konzept}

Als Versuch einer Antwort auf die drängenden Probleme wurde an der chirurgischen Klinik am Spital Bülach im April 2008 ein Pilotprojekt einer strukturierten WB für Studienabgänger in Gang gesetzt, welches das weite Spektrum der Anliegen der Assistenzärztinnen und Assistenzärzte aufnahm und gezielte Hilfeleistung anbot.

Voraussetzung des Projektes war die Schaffung einer Teilzeitstelle von zunächst $10 \%$ und später $20 \%$ 


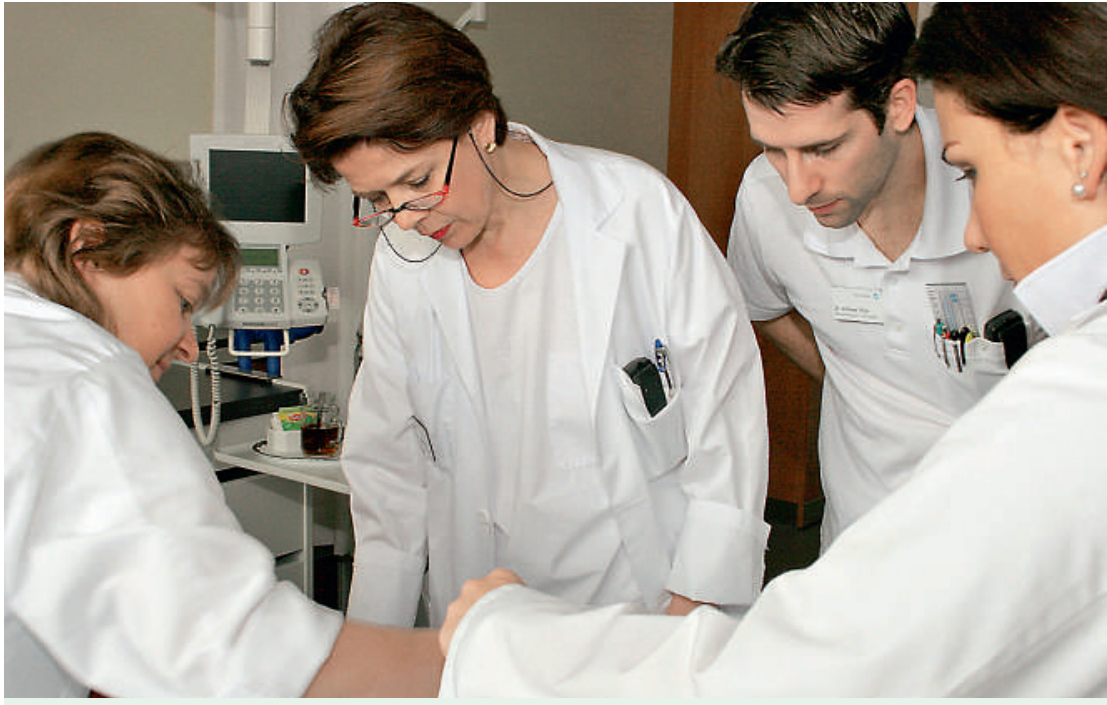

Weiterbildung ist jederzeit und überall möglich - wenn man daran denkt und sich Zeit nimmt.
- Individuelle Unterstützung beim Schliessen von fachlichen Lücken wie Therapiekonzepten, postoperativer Nachbehandlung, Lesen von Röntgenbildern.

- Besprechung der Schnittstellenproblematik im Dreieck Patient, Pflege und Assistenzärztin.

- Durchführung einer Down-to-Top-Umfrage mit der Software von Dr. med. M. Durban als Spiegel für das Kader.

Nicht im Pflichtenheft des LA in Weiterbildung stand die Assistenz im Operationssaal, da eine Teilzeitanstellung von $20 \%$ nicht ausreicht, sowie die individuelle Karriereplanung. Jede Assistenzärztin und jeder Assistenzarzt hat einen Tutor und innerhalb der Assistentenschaft einen Paten. Die Karriereplanung wird von der Chefärztin betreut. Der LA in Weiterbildung beeinflusst jedoch durch sein persönliches Coaching die Karriereplanung.

Der LA in WB muss selbst langjährige Erfahrung besitzen und von der Bedeutung seines Berufes überzeugt sein. Zudem muss er über professionelle Integrität verfügen und ein positives Rollenmodell verkörpern. Eine fundierte WB in Didaktik ist empfehlenswert. Vorteilhaft ist der Besuch eines Didaktikkurses, wie sie die Universitäten oder die ETH anbieten [6]. Ein Master of Medical Education (MME) ist für eine klinische Weiterbildungsstelle unserer Meinung nach nicht erforderlich.

Der LA in Weiterbildung für Assistenzärzte war ergänzender Teil des strukturierten Weiterbildungsund Rotationsprogrammes der Klinik. Dieses Programm umfasst seinerseits ein Feedbacksystem zur kontinuierlichen, persönlichen Optimierung der beruflichen Fähigkeit in Form regelmässiger Morbiditäts- und Mortalitätskonferenzen, transparente Einteilung zu Operationen und finanzielle und ideelle Unterstützung in externen Fortbildungsprogrammen.

\section{Resultat}

Als Bilanz des Pilotprojektes kann festgehalten werden, dass sich die jungen Assistenzärztinnen und Assistenzärzte durch diese Art Weiterbildung mit ständigem Feedback schneller und leichter im Arbeitsprozess zurechtfinden und lernen, ihre Aufgabe besser zu erfüllen. Gewisse Qualitätsstandards auf Visiten und in Rapporten konnten nachhaltiger verankert werden. Im Nebeneffekt verhilft diese Art Weiterbildung ähnlich einer Supervision zu einer besseren Betriebskultur.

Die Assistenzärztinnen und Assistenzärzte lernen neben dem chirurgischen Fach auch, sich besser in den Institutionen des Spitals und mit deren Regeln zurechtzufinden und Frustrationen zu ertragen. Sie lernen auseinanderzuhalten, welche Probleme personen- und welche institutsgebunden sind.

Strukturierte Weiterbildung ist ein wesentliches Mittel, die Attraktivität des Alltags der jungen Assistenzärzte zu steigern, Interesse zu wecken und die Motivabasierten medizinischen Grundsätzen. 
tion zu steigern, den Beruf des Chirurgen auszuüben und damit die Nachwuchslücke zu schmälern.

\section{Schlussfolgerung}

Welche Konsequenzen sind aus dem Pilotprojekt LA in Weiterbildung für Assistenzärzte zu ziehen?

Es sind nach einem klar definierten Raster der Grösse der Klinik entsprechende separate Stellenprozente LA in Weiterbildung zu schaffen, wie es die Pflege schon lange kennt und uns vorlebt.

Die für diese Stelle in Frage kommende Person muss didaktisch begabt sein, über fachliche Expertise verfügen, ein positives Rollenmodell darstellen und professionelle Integrität besitzen [7].

Der Auftrag lautet, an bestimmten Wochentagen Weiterbildung zu betreiben. In einem anderen Modell wäre es denkbar, an den definierten Wochentagen wechselnde Kaderpersonen einzusetzen. Diese Weiterbildung muss unantastbar reserviert sein, ansonsten sie Gefahr läuft, erodiert zu werden.

Das Pflichtenheft des Weiterbildners kann nach den Bedürfnissen der entsprechenden Klinik zusammengestellt werden. Es eignet sich vorzüglich zur Unterstützung des Einstiegs in das Berufsleben. Der Aufgabenkatalog des vorgestellten Pilotprojektes entsprach den Anliegen und Möglichkeiten der Klinik.

\section{Zur Nachahmung empfohlen ...}

Alle Massnahmen und Initiativen, die zum Ziel haben, die ärztliche Weiterbildung zu unterstützen, sind aus der Sicht des Schweizerischen Instituts für ärztliche Weiter- und Fortbildung SIWF hochwillkommen. Ein erfahrener Kaderarzt, der den Auftrag und genügend Zeit hat, die jungen Assistenzärztinnen und Assistenzärzte im Spital einzuführen und zu begleiten, kann ganz sicher viel dazu beitragen, dass sie sich schneller und besser an ihrem Arbeitsplatz zurechtfinden. In grösseren Kliniken hat der Weiterbildungsstättenleiter selber nicht immer genügend Zeit, sich den aktuellen und praktischen Problemen aller Ärzte in Weiterbildung anzunehmen, ihnen metho-
Die Tatsache, einen Weiterbildner mit vertraglich gesichertem Leistungsauftrag an der Klinik zu haben, wirkt sich positiv aus im Bemühen, interessierte und engagierte Assistenzärzte zu gewinnen. Sie ist eine gute Imagepflege.

Seien wir uns schliesslich bewusst: Von der erfolgreichen Weiterbildung in unserem Fachgebiet hängt die Zukunft unseres Berufes ab [2].

\section{Literatur}

1 Bauer H. Chirurgie in neuen Strukturen. Mitt Dtsch Ges Chir. 2007;338-47.

2 Walter U. Bleibt der Chirurg ein Beruf mit Zukunft? BDC. 2006;45:116-7.

3 Maio G. Heilen als industrieller Prozess? Der reine Markt als Gefährdung der ärztlichen Kernindentität. Schweiz Ärztezeitung. 2011;92(22):858-61.

4 Montagne S, Jucker-Kupper P, Berendonk C, Rogausch A, Beyeler C, Giger M. Drei Jahre Erfahrung mit Arbeitsplatz-basiertem Assessment (Mini-CEX und DOPS) in der ärztlichen Weiterbildung. Schweiz Ärztezeitung. 2001;91(4):109-11.

5 Dr. med. Michael Durband, Oberarzt, Chirurgische Klinik, Kantonsspital Aarau, 5100 Aarau.

6 Beispiele: www.hochschuldidakktik.uzh.ch oder www.didactica.ethc.ch

7 Muff B. Man muss lange leben, um ein Mensch zu werden. Schweiz Ärztezeitung. 2007;88(16):683.

dische Hilfestellung zu geben, Lücken in Wissen und Können «auszubügeln», Schnittstellen und deren Überbrückung zu zeigen oder regelmässig strukturiertes Feedback zu vermitteln.

Wie die Autoren mit Recht schreiben, hängen die Zukunft des Arztberufes und damit auch die Qualität des Gesundheitswesens zu einem entscheidenden Teil von der erfolgreichen Weiterbildung ab. Hoffentlich gibt das Bülacher Projekt Anstoss zu vielen weiteren Initiativen mit gleichem Ziel.

Dr. med. Werner Bauer, Präsident des Schweizerischen Instituts für ärztliche Weiter- und Fortbildung SIWF 